\title{
Cambios en la prevalencia de la caries dental en escolares de tres regiones de México: encuestas de 1987-1988 y de 1997-1998
}

\author{
Oscar Velázquez Monroy, ${ }_{1}^{1}$ Heriberto Vera Hermosillo, ${ }^{1}$ María Esther Irigoyen Camacho, ${ }^{2}$ \\ Adriana Mejía González ${ }^{1}$ y Teresa Leonor Sánchez Pérez ${ }^{2}$
}

RESUMEN Objetivo. Comparar la prevalencia y la gravedad de la caries dental en dos estudios de vigilancia epidemiológica realizados en tres regiones de México en los años 1987-1988 y en 1997-1998.

Métodos. La encuesta de 1987-1988 se aplicó a una muestra representativa de escolares de 6 a 10 años de edad de los estados de Nuevo León, Tabasco y el Distrito Federal (D.F.); adicionalmente se incluyeron escolares de 12 años del D.F. El total de alumnos examinados en 1987-1988 fue de 7590. En la encuesta de 1997-1998 se incluyó a escolares de 6 a 10 y 12 años de edad de las tres regiones estudiadas. El total de alumnos incluidos en 1997-1998 fue de 8 164. Se compararon las medias del indice de dientes cariados, perdidos u obturados (CPOD) de cada grupo de edad en ambos períodos.

Resultados. Los resultados mostraron una disminución de la prevalencia y de la gravedad de la caries dental en los grupos de edad examinados ( $<<0,05)$. En 1997-1998, tanto en el estado de Tabasco como en el de Nuevo León se logró la meta propuesta por la Organización Mundial de la Salud de reducir a no más de tres los dientes cariados, perdidos u obturados a los 12 años de edad, con indices de CPOD de 2,67 y 1,72, respectivamente, aunque el D.F. sobrepasó esa cifra tope $(C P O D=3,11)$.

Conclusión. La disminución observada en los índices CPOD podría deberse a varios factores, tales como el consumo de sal fluorada, el uso de dentífricos y enjuagues fluorados, y un mayor acceso a los servicios odontológicos.

Palabras clave Caries dental, salud bucal, fluoración, México.

En México, la prevalencia de la caries dental es elevada (1-3). Para mejorar el estado de salud bucal de la población, a mediados de los años ochenta se inició en el estado de México uno de los programas preventivos de mayor cobertura: la fluoración de la sal. En dicho estado, más de 11 millones de personas se

Secretaría de Salud, San Luis Potosí \#199, $9^{\circ}$ piso, Col. Roma CP 06700, México, D.F.

2 Universidad Autónoma Metropolitana-Xochimilco, México, D.F. benefician de esta medida (4). A principios de los años noventa, el programa se expandió al resto del país y actualmente tiene una cobertura cercana a los 80 millones de personas.

En 1995 se publicó en el Diario Oficial de la Federación la Norma Oficial Mexicana, en la cual se establece que la sal debe adicionarse con yodo y flúor en concentraciones de $30 \pm 10 \mathrm{mg}$ $\mathrm{I}^{-} / \mathrm{kg}^{3}$ y $250 \pm 50 \mathrm{mg} \quad \mathrm{F}^{-} / \mathrm{kg}^{4}$,

\footnotetext{
$\mathrm{I}^{-}=$ion yodo.

$4 \mathrm{~F}^{-}=$ion flúor
}

respectivamente, pudiéndose utilizar para la fluoración de la sal el fluoruro de sodio $(\mathrm{NaF})$ o el fluoruro de potasio (KF) (5). La norma determina que solamente la sal de consumo humano debe ser fluorada. Como parte de la vigilancia del Programa de Fluoración de la Sal, entre 1987 y 1988 se realizó un estudio epidemiológico que constituyó el inicio para la evaluación posterior del programa. Entre 1997 y 1998 se realizó una segunda encuesta epidemiológica. El objetivo del presente trabajo es comparar la 
prevalencia y gravedad de la caries dental en México según ambas encuestas (1987-1988 y 1997- 1998), específicamente en los estados de Tabasco y Nuevo León y en el Distrito Federal (D.F.).

\section{MATERIALES Y MÉTODOS}

\section{Población de estudio y muestreo}

La encuesta epidemiológica inicial (1987-1988) fue realizada en 11 de las 32 entidades federativas de México: Baja California Sur, Colima, Chiapas, México, Guerrero, Hidalgo, Morelos, Nuevo León, Tabasco, Yucatán y el Distrito Federal. Se seleccionaron niños de 5 a 10 años de edad que asistían a escuelas primarias y jardines de infancia públicos y privados incorporados a la Secretaría de Educación Pública (SEP). Únicamente en el D.F. se incluyó a escolares de 12 años de edad. En el presente trabajo se analizan los datos correspondientes a los escolares de 6 a 10 años de edad de los estados de Nuevo León y Tabasco, y de los escolares de 6 a 10 y 12 años de edad del D.F.

En la encuesta de 1987-1988 se utilizó un muestreo aleatorio en varias etapas. En la primera etapa de muestreo se elaboró una lista en que los municipios de cada estado se clasificaron como urbanos o rurales siguiendo la información del X Censo General de Población y Vivienda (6). La excepción fue el D.F., donde no se contempló ningún estrato rural y que para el muestreo se dividió en tres zonas geográficas: norte, centro y sur. De cada lista se seleccionó un número predeterminado de municipios siguiendo la técnica de probabilidad proporcional al tamaño, por lo que los municipios de mayor tamaño dentro de cada estado tuvieron una mayor probabilidad de ser seleccionados. Los municipios seleccionados constituyeron las unidades primarias de muestreo.

Asimismo, de la encuesta de 19871988 se excluyeron las escuelas ubicadas en localidades donde el agua de abastecimiento tuviera más de una parte por millón (ppm) de flúor debido a que el Programa de Fluoración de la Sal no abarca zonas con concentraciones de flúor superiores al límite permitido. También se excluyeron aquellas escuelas que tenían programas intensivos para la prevención de la caries dental que fueran diferentes de los ofrecidos al resto de la población escolar. En esta categoría se encontraban menos de $2 \%$ de las escuelas de los territorios estudiados.

En la segunda etapa de muestreo se seleccionaron las escuelas dentro de cada municipio de acuerdo con una lista proporcionada por la SEP. Dicha lista daba el número de niños por grado escolar y por edad. En cada municipio, el número de escuelas seleccionadas dependió del número de niños de 5 a 10 años de edad inscritos en Nuevo León y Tabasco, y el número de niños de 5 a 10 y 12 años de edad del D.F. La selección se hizo utilizando una probabilidad proporcional al tamaño de la escuela.

En la segunda encuesta también se diseño una muestra representativa de cada entidad federativa siguiendo un esquema similar al utilizado en la encuesta anterior (1987-1988). Esta segunda encuesta se inició en 1997 y se realizó de forma progresiva en todo el país. En Tabasco, Nuevo León y el D.F., los datos se recogieron entre 1997 y 1998, es decir, 10 años después de la primera encuesta y aproximadamente cuatro años después de haberse puesto en marcha en todo el país el Programa de Fluoración de la Sal. En este estudio se examinaron niños de 6 a 10 años de edad inscritos en las escuelas primarias y adolescentes de 12 años inscritos en las escuelas secundarias.

En esta segunda encuesta, a diferencia de la primera, se incluyó la concentración de flúor en el agua de abastecimiento como criterio de selección (7). Esto no afectó la selección del D.F. ni del estado de Tabasco, donde la mayor parte de las fuentes de abastecimiento de agua tenían menos de 0,3 ppm de flúor [F]. En el estado de Nuevo León, sin embargo, el intervalo de las concentraciones de flúor es más amplio y estas se agruparon en los siguientes estratos: bajo $([\mathrm{F}]<0,4$ ppm), medio $(0,4 \leq[\mathrm{F}]<0,7)$, óptimo $(0,7 \leq[\mathrm{F}] \leq 1,0$ ppm) y alto $([\mathrm{F}]>1$ ppm). El pozo con la mayor concentración de flúor tenía 1,5 ppm. La información de las concentraciones de flúor en el agua se obtuvo del Programa de Vigilancia de Fluoruros Naturales en el Agua para Consumo Humano, de la Secretaría de Salud (7). En el estado de Nuevo León, la asignación de escolares de 12 años se hizo tomando en cuenta la proporción de niños de esta edad en estratos, según la concentración de flúor en el agua, con el fin de garantizar la adecuada ponderación de la muestra y disminuir el riesgo de sesgos en el cálculo de los estimadores. Así, 37,0\% de los escolares seleccionados eran de localidades con pozos de abastecimiento con concentraciones bajas de flúor en el agua; 51,8\%, de localidades con concentraciones medias; 3,7\%, de localidades con concentraciones óptimas; y 7,4\%, de localidades con concentraciones altas.

Debe hacerse hincapié en que los niños de las escuelas primarias incluidas en la primera y segunda encuestas fueron seleccionados de las mismas escuelas. La selección de escuelas secundarias se hizo de novo, excepto en el D.F., donde se había recogido información acerca de este grupo de edad en el estudio inicial. Las regiones que se presentan en este informe (Nuevo León, Tabasco y D.F.) realizaron sus encuestas en el período otoño-invierno, tanto en la primera encuesta (1987-1988) como en la segunda (1997-1998).

\section{Criterio de diagnóstico y análisis}

En ambas encuestas se utilizó un protocolo utilizado por la Organización Mundial de la Salud (OMS) (8). Según este protocolo, el diagnóstico de la caries dental se hizo cuando una foseta, fisura o superficie lisa del diente presentaba el esmalte socavado con reblandecimiento en el piso o en cualquiera de las paredes de la lesión. Los examinadores fueron 
entrenados tanto en la forma en que se debía realizar la exploración de la cavidad bucal de los escolares como en el diagnóstico. Se siguió un programa cuidadoso para la estandarización de criterios y se contó con la colaboración de personal enviado por la OMS para tal propósito. En este programa se capacitó a 32 odontólogos para la realización de las encuestas. La concordancia inter e intraexaminador fue superior a $80 \%$ y $90 \%$, respectivamente.

Los examinadores utilizaron espejo y explorador, y los exámenes se hicieron con luz natural. Los datos se recogieron en formularios diseñados específicamente para el estudio. La información se introdujo en archivos electrónicos para su análisis. Con estos bancos de datos se calculó el número de dientes y de superficies dentarias cariadas, perdidas y obturadas (CPOD y CPOS, respectivamente), así como el porcentaje con que los componentes cariado $(\mathrm{C})$, perdido $(\mathrm{P})$ y obturado (O) contribuyen al índice total. También se calculó la prevalencia de caries dental en dentición permanente, definida como la proporción de escolares de la muestra con un índice CPOD mayor de 0 (CPOD $>0)$.

Mediante la prueba de $\chi^{2}$ de Pearson se compararon las prevalencias de caries dental en las dos encuestas. Asimismo, se utilizó la prueba estadística de la $t$ de Student para muestras independientes para comparar los índices de caries de ambas encuestas.

\section{RESULTADOS}

En la encuesta elaborada en 19871988 se incluyó a un total de 7590 alumnos de las tres regiones estudiadas, de los cuales 7223 tenían entre 6 y 10 años; en el caso del D.F. además de los niños de 6 a 10 años se examinaron 367 escolares de 12 años de edad. En la encuesta de 1997-1998 se incluyeron 8164 escolares de 6 a 10 años y de 12 años, provenientes de las mismas tres regiones estudiadas 10 años antes. En el cuadro 1 se presenta la distribución de la muestra por año de
CUADRO 1. Número de escolares evaluados por entidad federativa en las encuestas de 1987-1988 y de 1997-1998 en el Distrito Federal y los estados de Nuevo León y Tabasco, México

\begin{tabular}{|c|c|c|c|c|c|c|c|c|}
\hline \multirow{2}{*}{$\begin{array}{l}\text { Edad } \\
\text { (años) }\end{array}$} & \multicolumn{2}{|c|}{ Distrito Federal } & \multicolumn{2}{|c|}{ Tabasco } & \multicolumn{2}{|c|}{ Nuevo León } & \multicolumn{2}{|c|}{ Total } \\
\hline & $1987-88$ & $1997-98$ & 1987-88 & $1997-98$ & 1987-88 & $1997-98$ & $1987-88$ & 1997-98 \\
\hline $\begin{array}{l}6 \text { a } 10 \\
12\end{array}$ & $\begin{array}{r}3204 \\
367\end{array}$ & $\begin{array}{r}1594 \\
287\end{array}$ & $\begin{array}{r}1467 \\
0\end{array}$ & $\begin{array}{r}2102 \\
475\end{array}$ & $\begin{array}{r}2552 \\
0\end{array}$ & $\begin{array}{r}3290 \\
416\end{array}$ & $\begin{array}{r}7223 \\
367\end{array}$ & $\begin{array}{l}6986 \\
1178\end{array}$ \\
\hline Total & 3571 & 1881 & 1467 & 2577 & 2552 & 3706 & 7590 & 8164 \\
\hline
\end{tabular}

CUADRO 2. Caries en la dentición permanente de escolares del Distrito Federal, México, 1987-1988 y 1997-1998

\begin{tabular}{|c|c|c|c|c|}
\hline \multirow{2}{*}{$\begin{array}{l}\text { Edad } \\
\text { (años) }\end{array}$} & \multicolumn{2}{|c|}{ Escolares con caries } & \multicolumn{2}{|c|}{ Índice CPOD 1 (\%) } \\
\hline & 1987-88 & $1997-98$ & $1987-88$ & $1997-98$ \\
\hline 6 & 846 & 669 & $218(25,8)$ & $150(22,4)$ \\
\hline 7 & 865 & 259 & $429(49,6)$ & $98(37,8)$ \\
\hline 8 & 519 & 200 & $358(69,0)$ & $101(50,5)$ \\
\hline 9 & 509 & 231 & $400(78,6)$ & $128(55,4)$ \\
\hline 10 & 465 & 235 & $370(79,6)$ & $154(65,5)$ \\
\hline 12 & 367 & 287 & $324(88,3)$ & $228(79,4)$ \\
\hline Total & 3571 & 1881 & $2099(65,1)^{a}$ & $859(51,8)^{a}$ \\
\hline
\end{tabular}

CPOD: Índice de dientes cariados, perdidos y obturados.

ajustado por edad.

CUADRO 3. Caries en la dentición permanente de escolares del estado de Tabasco, México, $1987-1988$ y $1997-1998$

\begin{tabular}{|c|c|c|c|c|}
\hline \multirow{2}{*}{$\begin{array}{l}\text { Edad } \\
\text { (años) }\end{array}$} & \multicolumn{2}{|c|}{ Escolares con caries } & \multicolumn{2}{|c|}{ Índice CPOD 1 (\%) } \\
\hline & $1987-88$ & $1997-98$ & $1987-88$ & 1997-98 \\
\hline 6 & 262 & 401 & $122(46,6)$ & $40(10,0)$ \\
\hline 7 & 286 & 352 & $226(79,0)$ & $48(13,6)$ \\
\hline 8 & 290 & 519 & $250(86,2)$ & $112(21,6)$ \\
\hline 9 & 319 & 349 & $289(90,6)$ & $138(39,5)$ \\
\hline 10 & 310 & 481 & $287(92,6)$ & $213(44,3)$ \\
\hline 12 & - & 475 & - & $344(72,4)$ \\
\hline Total & 1467 & 2577 & $1174(65,8)^{a}$ & $895(33,6)^{a}$ \\
\hline
\end{tabular}

CPOD: Índice de dientes cariados, perdidos y obturados.

ajustado por edad.

CUADRO 4. Caries en la dentición permanente de escolares del estado de Nuevo León, México, 1987-1988 y 1997-1998

\begin{tabular}{|c|c|c|c|c|}
\hline \multirow{2}{*}{$\begin{array}{c}\text { Edad } \\
\text { (años) }\end{array}$} & \multicolumn{2}{|c|}{ Escolares con caries } & \multicolumn{2}{|c|}{ Índice CPOD $1(\%)$} \\
\hline & 1987-88 & 1997-98 & $1987-88$ & 1997-98 \\
\hline 6 & 1040 & 1284 & $149(14,3)$ & $92(7,2)$ \\
\hline 7 & 529 & 515 & $187(35,3)$ & $82(15,9)$ \\
\hline 8 & 330 & 521 & $177(53,6)$ & $126(24,2)$ \\
\hline 9 & 349 & 539 & $221(63,3)$ & $188(34,9)$ \\
\hline 10 & 304 & 431 & $209(68,8)$ & $162(37,6)$ \\
\hline 12 & - & 416 & - & $223(53,6)$ \\
\hline Total & 2552 & 3706 & $943(47,0)^{a}$ & $873(28,9)^{a}$ \\
\hline
\end{tabular}

CPOD: Índice de dientes cariados, perdidos y obturados.

ajustado por edad. 
estudio, entidad federativa y grupo de edad. El cuadro 2 muestra las prevalencias de la caries dental en el D.F., por grupos de edad en ambos estudios. Los cuadros 3 y 4 presentan estas mismas prevalencias en los estados de Tabasco y Nuevo León, respectivamente. La prevalencia más alta de caries dental en el estudio inicial se detectó en el estado de Tabasco. Dicha prevalencia disminuyó significativamente en todos los grupos de edad estudiados, según los datos obtenidos en la segunda encuesta (1997-1998) ( $P<0,01)$. En el D.F. también se encontraron reducciones significativas $(P<0,01)$ en la prevalencia de caries, aunque en menor escala (cuadro 2), en todos los grupos de edad menos en el de niños de 6 años $(P>0,05)$, mientras que en el estado de Nuevo León (cuadro 4) se observó una reducción significativa $(P<0,01)$ en todos los grupos de edad estudiados. Pese a esta disminución, todavía existe una elevada prevalencia de caries en los grupos estudiados, particularmente en el D.F., donde $79 \%$ de los escolares de 12 años presentaron caries dental en 1997-1998.

Los valores promedio del índice CPOD disminuyeron entre 1987-1988 y 1997-1998 en las tres entidades federativas estudiadas (cuadros 5-7). Esta disminución fue estadísticamente significativa en los diferentes grupos de edad estudiados $(P<0,05)$. Por ejemplo, en el D.F., los escolares de 12 años examinados en 1987-1988 tuvieron un índice CPOD promedio de 4,42, mientras que en 1997-1998 dicho promedio fue de 3,11. En el grupo de niños de 6 a 10 años, el índice CPOD se redujo 32,0\% entre ambos estudios en el D.F., 59,3\% en Nuevo León y 79,1\% en Tabasco.

Como se señaló anteriormente, la muestra de niños de 12 años del estado de Nuevo León de la encuesta de 19971998 se seleccionó teniendo en cuenta las concentraciones límite de flúor de los pozos de agua. Los resultados mostraron que en los pozos con [F] bajas, el índice CPOD promedio fue 1,84; en los pozos con [F] medias, 1,80; en los que tenían [F] óptimas, 1,68, y

CUADRO 5. Índice de dientes cariados, perdidos y obturados (CPOD) y su desviación estándar (DE), por edad, en los estudios de 1987-1988 y 1997-1998 en el Distrito Federal, México

\begin{tabular}{lcccccccc}
\hline \multirow{2}{*}{$\begin{array}{c}\text { Edad } \\
\text { (años) }\end{array}$} & \multicolumn{2}{c}{ Escolares con caries } & & \multicolumn{2}{c}{ Índice CPOD, (DE) } & & Reducción \\
\cline { 2 - 3 } & $1987-88$ & $1997-98$ & & $1987-88$ & $1997-98$ & & porcentual \\
\hline 6 & 846 & 669 & & 0,5 & $(1,0)$ & 0,4 & $(0,9)$ & 20,0 \\
7 & 865 & 259 & & 1,6 & $(1,5)$ & 0,83 & $(1,3)$ & 28,4 \\
8 & 519 & 200 & & 1,85 & $(1,7)$ & 1,32 & $(1,6)$ & 28,6 \\
9 & 509 & 231 & & 2,46 & $(1,9)$ & 1,51 & $(1,7)$ & 38,6 \\
10 & 465 & 235 & & 2,85 & $(2,2)$ & 1,94 & $(2,2)$ & 31,9 \\
$6-10$ & 3204 & 1594 & & $1,76^{\mathrm{a}}$ & $(1,7)$ & $1,20^{\mathrm{a}}$ & $(1,6)$ & 32,0 \\
12 & 367 & 287 & & 4,42 & $(3,2)$ & 3,11 & $(2,6)$ & 29,6 \\
\hline
\end{tabular}

ajustado por edad.

CUADRO 6. Índice de dientes cariados, perdidos y obturados (CPOD) y su desviación estándar (DE), por edad, en los estudios de 1987-1988 y 1997-1998 en el estado de Tabasco, México

\begin{tabular}{|c|c|c|c|c|c|c|c|}
\hline \multirow{2}{*}{$\begin{array}{l}\text { Edad } \\
\text { (años) }\end{array}$} & \multicolumn{2}{|c|}{ Escolares con caries } & \multicolumn{4}{|c|}{ Índice CPOD, (DE) } & \multirow{2}{*}{$\begin{array}{l}\text { Reducción } \\
\text { porcentual }\end{array}$} \\
\hline & $1987-88$ & $1997-98$ & 1987 & -88 & 199 & -98 & \\
\hline 6 & 262 & 401 & 1,11 & $(1,2)$ & 0,13 & $(0,5)$ & 88,2 \\
\hline 7 & 286 & 352 & 2,22 & $(1,2)$ & 0,20 & $(0,6)$ & 90,9 \\
\hline 8 & 290 & 519 & 2,87 & $(1,4)$ & 0,40 & $(0,9)$ & 86,0 \\
\hline 9 & 319 & 349 & 3,34 & $(1,7)$ & 0,94 & $(1,4)$ & 71,8 \\
\hline 10 & 310 & 481 & 4,15 & $(2,4)$ & 1,19 & $(1,7)$ & 71,3 \\
\hline $6-10$ & 1467 & 2102 & $2,74^{\mathrm{a}}$ & $(2,2)$ & $0,60^{\mathrm{a}}$ & $(1,7)$ & 79,1 \\
\hline 12 & - & 475 & - & - & 2,67 & $(2,8)$ & - \\
\hline
\end{tabular}

ajustado por edad.

CUADRO 7. Índice de dientes cariados, perdidos y obturados (CPOD) y su desviación estándar (DE), por edad, en los estudios de 1987-1988 y 1997-1998 en el estado de Nuevo León, México

\begin{tabular}{|c|c|c|c|c|c|c|c|}
\hline \multirow{2}{*}{$\begin{array}{l}\text { Edad } \\
\text { (años) }\end{array}$} & \multicolumn{2}{|c|}{ Escolares con caries } & \multicolumn{4}{|c|}{ Índice CPOD, (DE) } & \multirow{2}{*}{$\begin{array}{l}\text { Reducción } \\
\text { porcentual }\end{array}$} \\
\hline & $1987-88$ & $1997-98$ & 1987 & -88 & 199 & -98 & \\
\hline 6 & 1040 & 1284 & 0,26 & $(0,7)$ & 0,11 & $(0,5)$ & 57,6 \\
\hline 7 & 529 & 515 & 0,85 & $(1,2)$ & 0,29 & $(0,8)$ & 65,8 \\
\hline 8 & 330 & 521 & 1,32 & $(1,2)$ & 0,44 & $(0,9)$ & 66,6 \\
\hline 9 & 349 & 539 & 1,72 & $(1,3)$ & 0,8 & $(1,3)$ & 53,4 \\
\hline 10 & 304 & 431 & 2,04 & $(1,3)$ & 0,88 & $(1,4)$ & 56,8 \\
\hline $6-10$ & 2552 & 3290 & $1,24^{\mathrm{a}}$ & $(1,4)$ & $0,50^{\mathrm{a}}$ & $(1,4)$ & 59,3 \\
\hline 12 & - & 416 & - & & 1,72 & $(2,2)$ & - \\
\hline
\end{tabular}

a Ajustado por edad.

en aquellos con $[\mathrm{F}]$ altas el promedio fue $1,79(P>0,05)$.

La figura 1 muestra los valores promedios del CPOS en escolares de 6 a 10 años de edad de las encuestas de 1987-1988 y de 1997-1998. Como se esperaba, los resultados y los cambios entre 1987-1988 y 1997-1998 fueron similares a los del índice basado en el promedio de dientes afectados (CPOD).

Los cuadros 8-10 presentan la contribución porcentual relativa de 
FIGURA 1. Índice CPOS en escolares de seis a diez años* del Distrito Federal, Tabasco y Nuevo León en las encuestas de 1987-1988 y 1997-1998

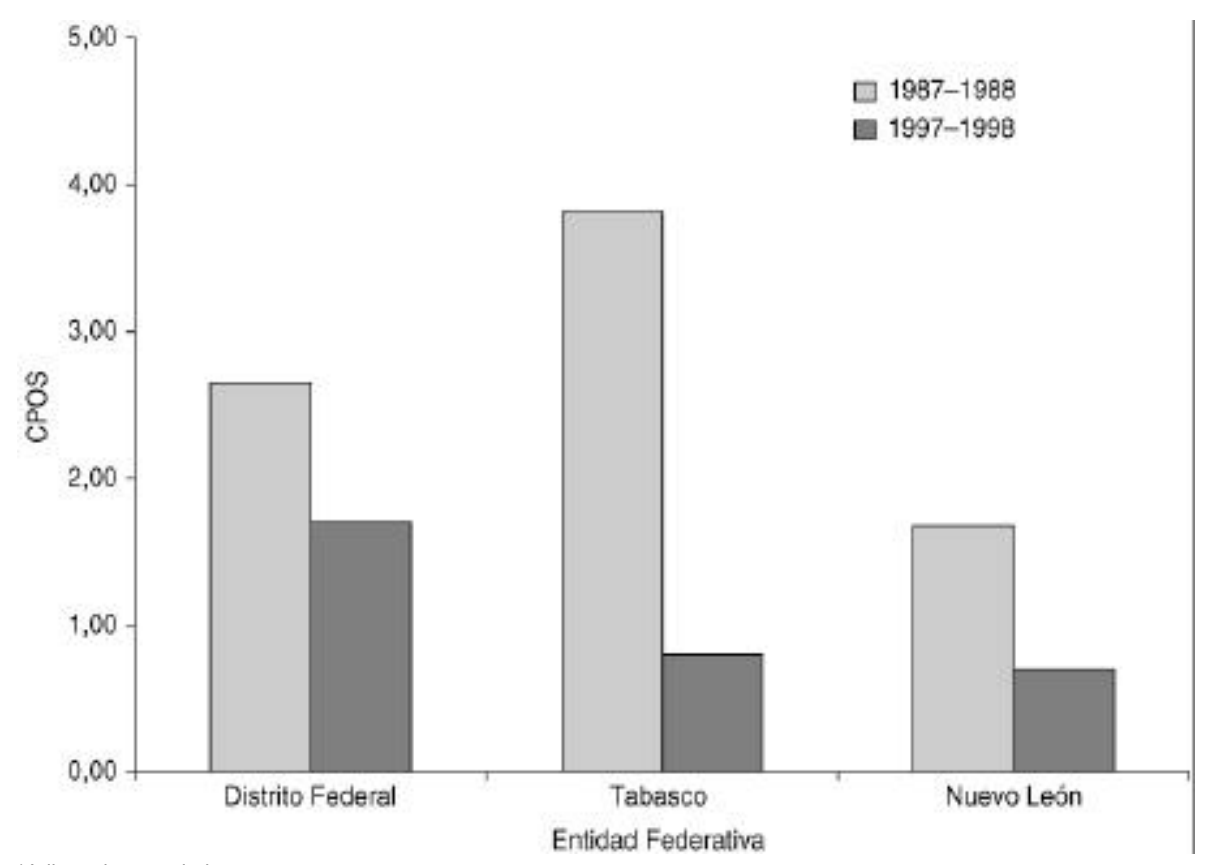

${ }^{*}$ Adjustado por edad

CUADRO 8. Contribuciones porcentuales relativas de las caries (C) y la obturación (0), por edad, en los estudios de 1987-1988 y 1997-1998 en el Distrito Federal, México

\begin{tabular}{|c|c|c|c|c|c|c|c|c|}
\hline \multirow{2}{*}{$\begin{array}{c}\text { Edad } \\
\text { (años) }\end{array}$} & \multicolumn{2}{|c|}{ Caries } & \multicolumn{2}{|c|}{ C/CPOD (\%) } & \multicolumn{2}{|c|}{ Obturación } & \multicolumn{2}{|c|}{ O/CPOD (\%) } \\
\hline & 1987-88 & 1997-98 & $1987-88$ & 1997-98 & 1987-88 & 1997-98 & 1987-88 & 1997-98 \\
\hline 6 & 0,47 & 0,38 & 96,0 & 95,0 & 0,02 & 0,02 & 4,1 & 5,0 \\
\hline 7 & 1,05 & 0,78 & 90,5 & 94,9 & 0,11 & 0,05 & 9,5 & 4,1 \\
\hline 8 & 1,60 & 1,23 & 86,5 & 93,2 & 0,25 & 0,09 & 13,5 & 6,8 \\
\hline 9 & 2,10 & 1,33 & 85,4 & 88,1 & 0,36 & 0,18 & 14,6 & 11,9 \\
\hline 10 & 2,23 & 1,56 & 78,2 & 80,4 & 0,61 & 0,38 & 21,4 & 20,6 \\
\hline $6-10$ & 1,49 & 1,06 & 84,7 & 88,0 & 0,27 & 0,14 & 15,3 & 12,0 \\
\hline 12 & 3,55 & 2,76 & 80,3 & 88,7 & 0,84 & 0,35 & 19,1 & 11,3 \\
\hline
\end{tabular}

CPOD: Índice de dientes cariados, perdidos y obturados.

CUADRO 9. Contribuciones porcentuales relativas de las caries (C) y la obturación (O), por edad, en los estudios de 1987-1988 y 1997-1998 en el estado de Tabasco, México

\begin{tabular}{|c|c|c|c|c|c|c|c|c|}
\hline \multirow{2}{*}{$\begin{array}{c}\text { Edad } \\
\text { (años) }\end{array}$} & \multicolumn{2}{|c|}{ Caries } & \multicolumn{2}{|c|}{ C/CPOD (\%) } & \multicolumn{2}{|c|}{ Obturación } & \multicolumn{2}{|c|}{ O/CPOD (\%) } \\
\hline & 1987-88 & $1997-98$ & $1987-88$ & 1997-98 & $1987-88$ & 1997-98 & 1987-88 & $1997-98$ \\
\hline 6 & 1,12 & 0,13 & 100 & 100 & 0 & 0 & 0 & 0 \\
\hline 7 & 2,21 & 0,17 & 99,5 & 85,0 & 0,01 & 0,02 & 0,5 & 10,5 \\
\hline 8 & 2,76 & 0,23 & 96,2 & 57,5 & 0,11 & 0,16 & 3,8 & 40,0 \\
\hline 9 & 3,14 & 0,46 & 94,0 & 49,0 & 0,19 & 0,46 & 5,7 & 49,0 \\
\hline 10 & 3,92 & 0,50 & 94,2 & 42,0 & 0,22 & 0,67 & 5,3 & 56,3 \\
\hline $6-10$ & 2,63 & 0,30 & 96,0 & 52,3 & 0,11 & 0,26 & 3,9 & 46,0 \\
\hline 12 & - & 1,33 & - & 49,8 & - & 1,23 & - & 46,1 \\
\hline
\end{tabular}

CPOD: Índice de dientes cariados, perdidos y obturados. cada uno de los elementos del CPOD para el D.F. y los estados de Tabasco y Nuevo León. En ambos estudios, el elemento del índice CPOD con la más alta representación porcentual fue el número de dientes cariados (C). En el estudio inicial, el porcentaje fue superior a $78 \%$ en las tres entidades federativas estudiadas, mientras que en los datos de 1997-1998, se observó que el componente de dientes con caries (C) seguía teniendo el valor más alto. Sin embargo, en el estado de Tabasco hubo un aumento importante del componente de obturación (O), acompañado de una disminución de la proporción que ocupa en el índice el componente de caries.

\section{DISCUSIÓN}

\section{Tendencias}

Los resultados del presente estudio revelan una reducción de la prevalencia y gravedad de la caries dental en la dentición permanente de niños de 6 a 10 años en la población estudiada. Tanto en Europa como en países de la región americana con programas de fluoración de la sal se han observado reducciones importantes de la prevalencia de la caries dental (9-13).

La reducción observada en los niños estudiados en México puede deberse a varios factores. En primer término, la población en esas tres entidades federativas ha consumido sal fluorada por un mínimo de cuatro años. Los datos obtenidos mediante la vigilancia de la concentración de fluoruro en la sal por parte de la Dirección General de Calidad Sanitaria de Bienes y Servicios (14) y de investigadores independientes (15) han mostrado concentraciones de fluoruro por debajo de lo señalado en la Norma Oficial Mexicana. Así, en el D.F., $60 \%$ de la sal contenía concentraciones menores de $200 \mathrm{ppm}$ en 1995. Muestreos recientes indican que la industria salinera ha mejorado la dosificación de flúor, lo que ha redundado en un mejor cumplimiento de lo especificado en la Norma Oficial. 
CUADRO 10. Contribuciones porcentuales relativas de las caries (C) y la obturación (0), por edad, en los estudios de 1987-1988 y 1997-1998 en el estado de Nuevo León, México

\begin{tabular}{|c|c|c|c|c|c|c|c|c|}
\hline \multirow{2}{*}{$\begin{array}{l}\text { Edad } \\
\text { (años) }\end{array}$} & \multicolumn{2}{|c|}{ Caries } & \multicolumn{2}{|c|}{ C/CPOD (\%) } & \multicolumn{2}{|c|}{ Obturación } & \multicolumn{2}{|c|}{ O/CPOD (\%) } \\
\hline & $1987-88$ & $1997-98$ & 1987-88 & $1997-98$ & $1987-88$ & $1997-98$ & $1987-88$ & $1997-98$ \\
\hline 6 & 0,25 & 0,11 & 96,3 & 100 & 0,01 & 0 & 3,8 & 0 \\
\hline 7 & 0,82 & 0,28 & 96,5 & 96,6 & 0,03 & 0,01 & 3,5 & 3,4 \\
\hline 8 & 1,25 & 0,38 & 94,7 & 86,4 & 0,07 & 0,06 & 5,3 & 13,6 \\
\hline 9 & 1,61 & 0,66 & 94,2 & 82,5 & 0,09 & 0,13 & 5,3 & 16,3 \\
\hline 10 & 1,76 & 0,68 & 86,3 & 77,3 & 0,28 & 0,20 & 13,7 & 22,7 \\
\hline $6-10$ & 1,14 & 0,42 & 91,8 & 84,4 & 0,10 & 0,08 & 7,7 & 16,0 \\
\hline 12 & - & 1,24 & - & 71,5 & - & 0,48 & - & 27,9 \\
\hline
\end{tabular}

CPOD: Índice de dientes cariados, perdidos y obturados.

Dados los problemas de dosificación antes mencionados, es probable que el efecto preventivo del fluoruro no haya sido el óptimo.

Es probable que parte de la reducción de la prevalencia y de la gravedad de la caries dental sea el resultado de la utilización de dentífricos fluorados. De acuerdo con los datos de la Encuesta Nacional de Ingresos y Gastos, realizada por el Instituto Nacional de Geografía e Informática, el porcentaje de familias que compran dentífricos ha aumentado en México, de $68,8 \%$ en 1989 a $79,1 \%$ en 1998 (16, 17). Asimismo, en el período entre los años 1989 y 2000 se notificó un incremento de la producción de dentífricos: en 1989 se fabricaron 25441 toneladas y en 2000, 41879 toneladas $(18,19)$. Un estudio longitudinal realizado en escolares del D.F. mostró una asociación entre la utilización de dentífricos fluorados y los índices de caries (20). Asimismo, a estos productos se les atribuye la reducción de la caries dental observada en países sin programas nacionales preventivos a base de fluoruros sistémicos (21). Asimismo, debe considerarse que en los estados encuestados y en el D.F. hay programas escolares preventivos para la caries dental que se basan en el uso de colutorios con fluoruros (22).

\section{Prevalencia y gravedad}

En 1987-1988, la prevalencia y la gravedad de la caries dental fueron mayores en Tabasco, seguido por el D.F. y el estado de Nuevo León. En 1997-1998, el D.F. presentó la prevalencia y gravedad más altas, seguido de Tabasco y Nuevo León. Adicionalmente, en 1997-1998, tanto en Tabasco como en Nuevo León se alcanzó la meta de la OMS (CPOD $\leq 3$ en niños de 12 años). El D.F. estuvo muy cerca de alcanzar dicha meta y es probable que, si la tendencia descendente continúa, la meta sea alcanzada en los próximos años.

Tanto en 1987-1988 como en 19971998, el componente porcentual más alto en el CPOD fue el de dientes con caries (C): más de 78\% en Nuevo León y en el D.F. La excepción fue el estado de Tabasco, donde en 1997-1998 el porcentaje de dientes obturados ascendió a más de $40 \%$ en varios grupos de edad. En lo que respecta al personal de salud, los datos del Sistema Nacional de Salud indican que la proporción de odontólogos es ligeramente superior en Tabasco $(9,4 \%)$, seguido del D.F. $(6,6 \%)$ y de Nuevo León (5,0\%). Asimismo, en Tabasco se notificaron 101 consultas dentales por 1000 habitantes, seguido por el D.F. con 41,8 consultas y Nuevo León con 39,6, lo que probablemente explicaría la mayor proporción de dientes obturados que se registró en Tabasco durante la segunda encuesta (22).

\section{Limitaciones}

En las encuestas analizadas en el presente estudio se utilizó el mismo método y el mismo criterio de diagnóstico (el de la OMS). No obstante, es posible que exista cierta diferencia en la interpretación y la aplicación de dicho criterio, sobre todo después de un período de 10 años y con un numeroso grupo de examinadores. Por lo tanto, es factible que el porcentaje de reducción en la población puedan ser distintos de los estimados en el presente estudio. Sin embargo, como ya se ha señalado, para disminuir la posibilidad de sesgo participaron, en la medida de lo posible, los mismos examinadores en ambos estudios y se seleccionaron las mismas escuelas. Si se considera que no hay una distribución diferencial por categorías de enfermedad en los alumnos matriculados en las escuelas creadas después de 1987-1988, es probable que esto haya disminuido el riesgo de un sesgo diferencial al comparar los datos de ambos estudios.

\section{CONCLUSIONES}

En México se detectó una disminución en los índices de caries dental en las entidades federativas de Tabasco, Nuevo León y el D.F. Las razones que explican dicha disminución son varias, desde el consumo de sal fluorada hasta un mayor acceso a la atención odontológica. Es importante insistir en la necesidad de reforzar la vigilancia química y epidemiológica del Programa de Fluoración de la Sal en México, con el fin de garantizar los beneficios de esta medida preventiva para la población.

Agradecimientos. Se agradece a la Organización Panamericana de la Salud, al Centro de Colaboración de la Organización Mundial de la Salud en San Antonio, a la fundación W. K. Kellogg y a los Centros para el Control y Prevención de Enfermedades, de Georgia, Estados Unidos, el apoyo brindado durante la realización del presente estudio. Asimismo, agradecemos a la Industria Salinera Mexicana su colaboración con el Programa de Fluoración de la Sal en México. 


\section{REFERENCIAS}

1. Duffo S, Maupome HG, Diez de Bonilla H, Hernandez Guerrero JC. Caries experience in a selected patient population in Mexico City. Community Dent Oral Epidemiol 1996;24: 298-289.

2. Gonzalez M, Cabrera R, Grossi SG, Franco F, Aguirre A. Prevalence of dental caries and gingivitis in a population of Mexican schoolchildren. Community Dent Oral Epidemiol 1992;21:11-14.

3. Irigoyen M, Szpunar S. Dental caries status of 12-year-old students in the State of Mexico. Community Dent Oral Epidemiol 1994;22: 311-314.

4. Irigoyen ME, Sánchez-Hinojosa S. Changes in dental caries prevalence in 12-year-old students in the State of Mexico after 9 years of salt fluoridation. Caries Res 2000;34:303-307.

5. Secretaría de Salud. NOM-040 SSA1-1993. Bienes y Servicios, sal yodatada y sal yodatada y fluorada. Especificaciones sanitarias. Diario Oficial, 13 de marzo de 1995.

6. Instituto Nacional de Estadística, Geografía e Informática. X Censo General de Población y Vivienda 1980, Resumen general abreviado. México, D.F.: INEGI; 1986.

7. Secretaría de Salud, Centro Nacional de Vigilancia Epidemiológica. Monitoreo de fluoruros naturales en agua para consumo humano, 1988-2001. México, D.F.: SSACNVE; 2002.

8. World Health Organization. Oral health surveys, basic methods. Geneva: WHO; 1987.

9. Toth K. A study of 8 years of domestic salt fluoridation for prevention of caries.
Community Dent Oral Epidemiol 1976;4:106-110.

10. Cahen PM, Turlot JC, Frank RM, OrbyMusset AM. National survey of caries prevalence in 6-15-year-old children in France. J Dent Res 1989;68:64-68.

11. Estupiñan-Day $S R$, Baez $R$, Horowitz $H$, Warpeha R, Sutherland B, Thamer M. Salt fluoridation in Jamaica. Community Dent Oral Epidemiol 2001 Aug;29(4):247-252.

12. Salas-Pereira M, Solano S. La fluoración de la sal en Costa Rica y su impacto en la caries dental. Fluoración al día 1994:4:13-19.

13. Solorzano I, Beltran E, Salas M, Chavarria S, Estupiñan S. Prevalence and trends of dental caries in Costa Rican school children from 1984 to 1999 [Abstract No. 5]. J Dent Res 2001; 80(Spec Iss):36.

14. Dirección General de Calidad Sanitaria de Bienes y Servicios, Programa de Vigilancia Sanitaria de la Sal. Comportamiento sanitario de la sal con respecto a la adición de flúor. México, D.F.: DGCSBS; 2000.

15. Maupomé G, Jaramillo R, Andrade LC, Juárez PL, López PR, Sánchez NW, et al. Flúor contenido en la sal para consumo humano distribuida en la Ciudad de México. Bol Oficina Sanit Panam 1995;119(3):195-201.

16. Instituto Nacional de Estadística, Geografía e Informática. Encuesta Nacional de Ingresos y Gastos de los Hogares (ENIGH), 1989. México, Ags.: INEGI; 1992.

17. Instituto Nacional de Estadística, Geografía e Informática. Encuesta Nacional de Ingresos y Gastos de los Hogares (ENIGH), 1998. México, Ags.: INEGI; 2000.
18. Instituto Nacional de Estadística, Geografía e Informática. Encuesta Industrial Mensual, resumen anual, 1989. México, Ags.: INEGI; 1991.

19. Instituto Nacional de Estadística, Geografía e Informática. Encuesta Industrial Mensual, resumen anual, 2000. México, Ags.: INEGI; 2001.

20. Irigoyen ME, Zepeda MA, Sánchez L, Molina N. Prevalencia e incidencia de caries dental y hábitos de higiene bucal en un grupo de escolares del sur de la Ciudad de México: estudio de seguimiento longitudinal. Rev Asoc Dent Mex 2001;53:98-104.

21. Haugejorden O, Nord A, Klock KS. Direct evidence concerning the major role of fluoride dentifrice in the caries decline. Acta Odontol Scand 1997:55:173-180.

22. Secretaría de Salud, Dirección General de Informática y Evaluación del Desempeño. Sistema de Información en Salud para la Población Abierta. Atención estomatológica: México, enero-diciembre de 1998. México, D.F.: SSA-DGIED; 1999.

Manuscrito recibido el 26 de marzo de 2002. Aceptado para publicación, tras revisión, el 17 de enero de 2003.

ABSTRACT Objective. To compare the prevalence and severity of dental caries found in epidemiological surveillance studies conducted in three areas of Mexico in two different periods, 1987-1988 and 1997-1998.

\section{Changes in the prevalence of dental caries in schoolchildren in three regions of Mexico: surveys from 1987-1988 and 1997-1998}

Methods. The 1987-1988 survey was done with representative samples of schoolchildren 6 to 10 years old in two states, Nuevo León and Tabasco, as well as in the country's Federal District (which includes Mexico City). The Federal District's 1987- 1988 sample also included schoolchildren who were 12 years old. The total number of students examined in the 1987-1988 period was 7590 . The 1997-1998 survey included schoolchildren who were 6-10 years old and 12 years old, in all three of the areas. The total number of students included in the 1997-1998 survey was 8164 . To compare the prevalence and severity of dental caries in the two periods, the average value for the index of decayed, missing, and filled teeth (DMFT) was used with all of the age groups.

Results. The values of the DMFT indices were significantly lower in the 1997-1998 period for all of the age groups studied ( $\mathrm{P}<0.05$ for all the age groups). In the 19971998 period both Tabasco and Nuevo León achieved the goal recommended by the World Health Organization of an average of no more than three decayed, missing, and filled teeth among the 12-year-olds, with DMFT indices of 2.67 and 1.72, respectively. However, in the Federal District in that 1997-1998 period the DMFT index exceeded the recommended level, with a value of 3.11.

Conclusions. The reduction seen in the DMFT indices could be due to several factors, including the consumption of fluoridated salt, the use of dentifrices and fluoride rinses, and broader access to dental services. 